UCRL- 102980

PREPRINT

\title{
APPLICATION OF KINETIC INDUCTANCE THERMOMETERS TO X-RAY CALORIMETRY
}

\author{
Y.C. Wai, S.E. Labov, and E.H. Silver \\ High Temperature Physics Division \\ Lawrence Livermore National Laboratory \\ Livermore, CA 94550
}

SPIE's 1990 International Symposium on

Optical and Optoelectronic Applied Science and Engineering

San Diego, CA, 7-13 July 1990

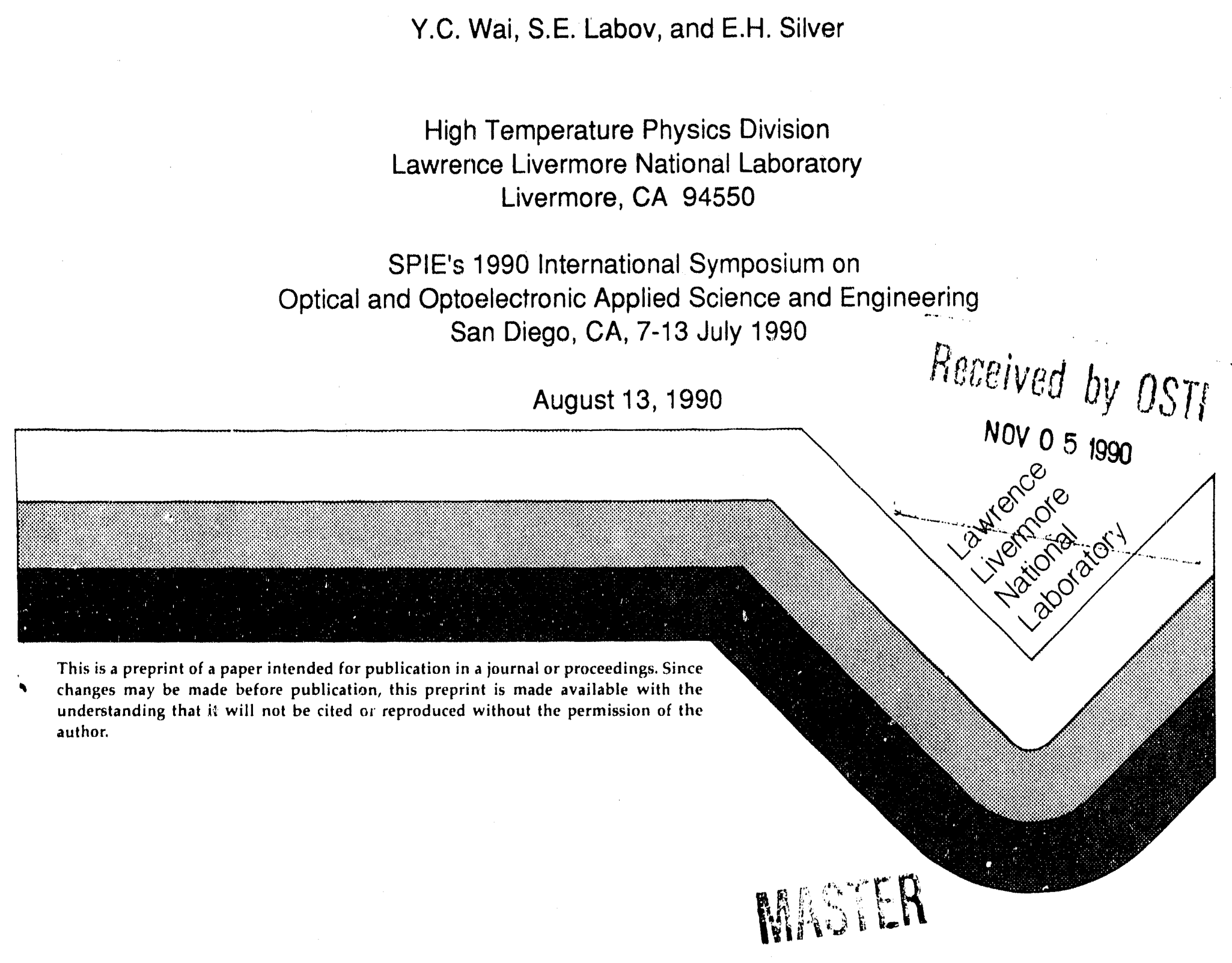

This is a preprint of a paper intended for publication in a journal or proceedings. Since changes may be made before publication, this preprint is made available with the understanding that is will not be cited or reproduced without the permission of the author. 
DISCI.AIMER

This document was prepared as an account of work spensored by an agency of the I Inited States Government. Neither the United States Government nor the Uriversity of C'alifornia nor any of their employees, makes any warranty, express or implied, or assumes any legal liability or responsibility for the accuracy, completeness, or usefulness of any information, apparatus, product, or process disclosed, or represents that its use would not infringe privately owned rights. Reference herein 10 any specific commercial products, pricess, or service by trade name, trademark, manufacturer, or otherwise, diess not necessarily constitute or imply its endorsement, recommendation, or favoring by the United States Government or the I Iniversity of Califurnia. The views and opinions of authors expressed herein do not necessarily state or reflect those of the United States Government or the University of California, and shall not be used for advertising or product endorsement purposes. 


\title{
Application of kinetic inductance thermometers to $\mathrm{x}$-ray calorimetry
}

\author{
Yolanda C. Wai, Simon E. Labov and Eric H. Silver \\ Laboratory for Experimental Astrophysics \\ Lawrence Livermore National Laboratory \\ UCRL -102980 \\ DE91 001768
}

\begin{abstract}
A kinetic inductance thermometer is applied to $\mathrm{x}$-ray calorimetry, and its operation over a wide range of frequencies and geometries is discussed. Three amplifier configurations are described, one using a superconducting quantum interference device (SQUID) amplifier, another incorporating an FET amplifier in an amplitude modulated system, and the third, using a tunnel diode frequency modulated oscillator circuit. The predicted performance of each configuration is presented.
\end{abstract}

\section{INTRODUCTION}

We are interested in fabricating broad band $x$-ray detectors operating between 0.1 and $10 \mathrm{keV}$ with the highest achievable energy resolution. In principle, $x$-ray microcalorimeters operating at cryogenic temperatures should be able to cover this energy band with a $1 \mathrm{eV}$ resolution. ${ }^{1,2}$ To date, $12 \mathrm{eV}$ has been achieved using resistive sensors thermally connected to an $x$-ray aosorbing material. ${ }^{3}$ In these devices, the resistive Johnson noise of the sensor is always present and is a limitation to the overall performance. To further improve the resolution of the device, the Johnson noise must be reduced. If the Johnson and amplifier noise can b reduced, the temperature fluctuation noise will dominate the noise spectrum over a much larger range in frequencies. Experiments are clurrently being carried out with calorimeiers using dielectric sensors. ${ }^{4}$ In principle, dielectric calorimeters are free of Johnson noise, and produce signal strengths far above the remaining amplifier noise. Another way to circurnvent Johnson noise is through the use of superconducting materials. Below the critical temperature, only the residual resistance from superconducting mirfaces need to be considered, and they prove to be negligible. ${ }^{5}$ As suggested by McDonald, ${ }^{6}$ the temperature dependence of the kinetic inductance in a superconducting thin film can be utilized as a thermal sensor. Work has been initiated to fabricate kinetic inductance $x$-ray calorimeters, ${ }^{7}$ but there has not been a discussion in the literature that describes how best to apply kinetic inductance thermometry to $x$-ray calorinetry. We describe here several techniques for operating kinetic inductance $x$-ray calorimeters.

We begin in Section 2 by summarizing the general properties of kinetic inductance. Section 3 discusses the parameters of a hypothetical detector which we propose as a prototype kinetic inductance $\mathrm{x}$-ray calorimeter. In Section 4 we predict the performance of the calorimeter when used with various amplifier configurations. These include a SQUID amplifying system, an amplitude modulated system which uses an FET amplifier, and a tunnel diode frequency modulated readout scheme. We conclude in Section 5 with an appraisal of the predicted performance of each configuration discussed, and the merits and disadvantages of each one.

\section{THE KINETIC INDUCTANCE THERMOMETER}

We describe a thermat sensor which makes use of the temperature dependent kinetic inductance of a thin-film superconductor. Kinetic inductance arises from the inertial mass of the Cooper pairs in a superconductor. The Cooper pair density varies with temperature, hence, the $k$ inetic inductance also has a temperature dependence. The inductive sensor consists of a superconducting stripline insulated from a groundplane by a thin dielectric layer; such a configuration behaves as a finite transmission line. At frequencies that are small compared to $1 / \sqrt{L C}$, where $L$ and $\mathrm{C}$ are the total inductance and electrical capacitance of the device, the impedance is strictly inductive.

In general, the tutal inductance for the device is

$$
L_{\text {total }}=\mu_{o}\left(\frac{l}{w}\right)_{s t}\left[d_{d i} \mu_{r}+\lambda_{s t} \operatorname{coth}\left(\frac{d_{s t}}{\lambda_{s t}}\right)+\lambda_{g p} \operatorname{coth}\left(\frac{d_{g p}}{\lambda_{g p}}\right)\right]
$$


Here, the subscripts $d i$, st and $g p$ refer to the dielectric, stripline and groundplane components, respectively. The thickness of each component is $d, \lambda$ is the penetration depth, $\mu_{0}$ is the magnetic permeability of free space, and $\mu_{r}$ is the relative magnetic permealiucy in the dielectric. The total length and width of the stripline are $l$ and $w$, respectively.

Equation 1 represents a sum of the magnetic inductance and the kinetic inductances of the stripline and the groundplane. The dimensions of the stripline and groundplane are chosen such that $d_{g p} \ll<\lambda_{g p}$, and $d_{s t} \gg \lambda_{s t}$. In this case, the stripline kinetic inductarice becomes negligible compared to the groundplane kinetic inductance. If the thickness of the dielectric is made sufficiently small, the magnetic inductance (the first term in Equation 1) will not contribute to the total inductance. In this limit, the inductance reduces to

$$
L_{k}(T)=\mu_{o}\left(\frac{l}{w}\right)_{s t} \frac{\lambda(T)_{g p}^{2}}{d_{g p}} .
$$

Here, $\lambda(T)$ is the temperature dependent penetration depth of the groundplane. The explicit temperature dependence of the penetration depth is based on the two fluid model: ${ }^{8}$

$$
\lambda(T)=\frac{\lambda_{o}}{\sqrt{\left(1-(T / T C)^{4}\right)}}
$$

where $\lambda_{0}$ is the penetration length of the superconducting material at $0 \mathrm{~K}$ and $T_{c}$ is the critical temperature of the groundplane material. In the readout configurations considered here, a large $(l / w)_{s t}$ ratio is used to increace $L_{k}$ and hence the signal to amplifier noise ratio of the sensor.

\section{CHARACTERISTICS OF THE PROPOSED DEVICES}

As shown in Equation 3, the kinetic inductance has a temperature dependence of the form $\left[1-\left(T / T_{c}\right)^{4}\right]^{-1}$ where the operating temperature, $T$, must not exceed $T_{c}$. As we will see in Section 4 , the dependence of the signal on $L_{k}$ is a function of the specific amplifier/readout circuit scheme. In all cases, the signal height is maximized if the temperature is kept as close to $T_{c}$ as possible. The optimum operating temperature depends on three coupled parameters, the heat capacity of the device, the maximum $x$-ray energy to be studied, and the desired energy resolution. The device featured here is designed to measure $x$-rays with energies up to $10 \mathrm{keV}$; the base temperature, therefore, must be chosen so that a $10 \mathrm{keV} x$-ray will not raise the temperature above the critical temperature. The heat capacity determines this temperature excursion and can be calculated from the expression for the energy resolution in the phonion noise limit $\delta E_{\text {phomon }}(F W H M)=2.35 \sqrt{k_{B} T^{2} C_{v}}$. Here $s_{B}$ is the Boltzmann constanth and $C_{v}$ is the total heat capacity of the device. For a calorimeter with a resolution of $1 \mathrm{eV}$ ofrrating in the phonon noise limit, the heat capacity of the device at a base temperature of $100 \mathrm{mK}$ is $3.4 \times 10^{-14} \mathrm{~J} / \mathrm{K}$. Accounting for the temperature dependence of the heat capacity $\left(C_{v} \propto T^{3}\right)$, the change in temperature due to absorption of a $10 \mathrm{keV} \times$-ray is then $25 \mathrm{mK}$. Such a large temperature excursion means that the base temperature of the device must be held below $0.75 T_{c}$. At this ternperature, the penetration depth (Equation 3) changes slowly with temperature. For most $\mathrm{x}$-ray energies, the sensor signal will be much smaller than it would be with a device operating closer to the critical temperature. Therefore, rather than pressing for a resolution of $1 \mathrm{eV}$, the device featured here has about ten times more heat capacity, and a theoretical resolution of $3 \mathrm{eV}$. The resulting temperature excursion for a $10 \mathrm{keV}$ photon is then reduced to $6 \mathrm{mK}$, allowing the operating temperature to shift from $0.75 T_{c}$ to $0.95 T_{c}$. The larger heat capacity also allows the detector to have a larger area. That permits a bigger $1 / w$ ratio of the stripline, and consequently, a larger kinetic inductance. The resulting sensor will have an improved signal to amplifier noise ratio, and will be more likely to achieve its $3 \mathrm{eV}$ phonon noise limit.

Our hypothetical detector, as shown in Figure 1, uses iridium as the superconducting groundplane with a $T_{c}=$ $0.11 \mathrm{~K} .{ }^{9}$ To operate in the kinetic inductance limit, we choose in iridium groundplane thickness of $4 \mathrm{~nm}$. This thickness is $10 \%$ of the minimum penetration depth. The iridium is evaporated onto a diamond substrate, followed by a silicon monoxide dielectric layer and then a niobium stripline. This detector system can be fabricated with a variety of $x$-ray absorber materials. 


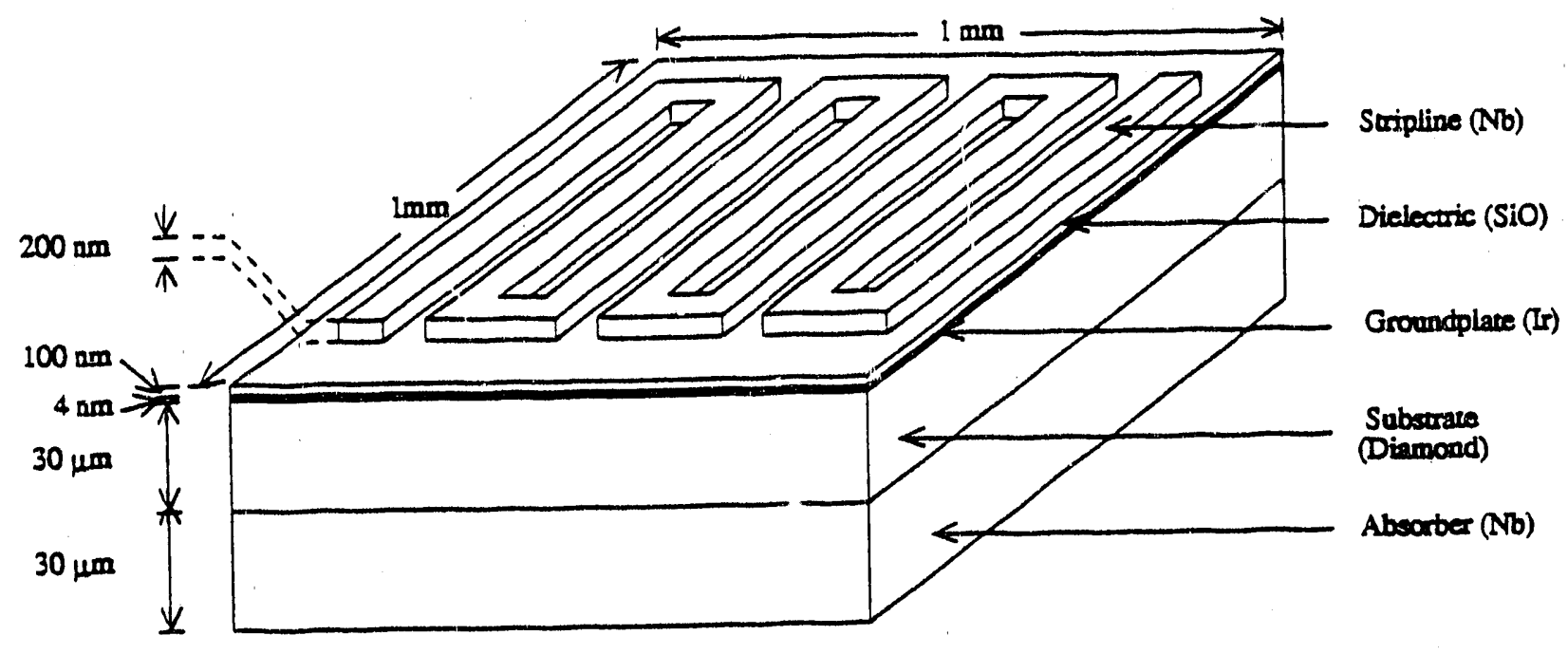

Figure 1. Schematic of the proposed $3 e \mathrm{~V}$ detector.

The thin film thermometer can be applied directly to a bulk absorber, or to a substrate with an absorber deposited on the opposite side. ${ }^{2}$ Possible $\mathrm{x}$-ray absorbers include low band gap materials such as $\mathrm{HgCdTe}$, $\mathrm{HgTe}$, or superconductors such as $\mathrm{Nb}$ or $\mathrm{Sn}$. We have chosen $30 \mu \mathrm{m}$ of niobium for this example because it is easy to fabricate. Its absorption efficiency at $10 \mathrm{keV}$ is $84 \%$ and almost $100 \%$ at $6 \mathrm{keV}$. The dimensions $(l \times w \times d)$ of the $3 \mathrm{eV}$ detector operating at $0.95 T_{c}$, together with the maximum heat capacity contributions from each component are given in Table 1 below:

\section{TABLE 1}

Hypothetical Kinetic Inductance Calorimeter with a 3 cV Energy Resolution

$$
\text { Components Dimensions } \quad C_{\mathrm{v}}(J / K)
$$

\begin{tabular}{lcc}
\hline \hline Stripline (Nb) & $16 \mathrm{~cm} \times 2 \mu m \times 200 \mathrm{~nm}$ & $7.87 \times 10^{-16}$ \\
Dielectric (SiO) & $1 \mathrm{~mm} \times 1 \mathrm{~mm} \times 100 \mathrm{~nm}$ & $8.54 \times 10^{-17}$ \\
Groundplane(Ir) & $1 \mathrm{~mm} \times 1 \mathrm{~mm} \times 4 \mathrm{~nm}$ & $3.83 \times 10^{-14}$ \\
Substrate (Diamond) & $1 \mathrm{~mm} \times 1 \mathrm{~mm} \times 30 \mu \mathrm{m}$ & $2.14 \times 10^{-15}$ \\
Absorber(Nb) & $1 \mathrm{~mm} \times 1 \mathrm{~mm} \times 30 \mu \mathrm{m}$ & $3.69 \times 10^{-13}$ \\
\end{tabular}




\section{AMPLIFIER CONFIGURATIONS}

A variety of readout schemes are possible for the devices described above, each transforming the temperature changes to useful electronic signals. We discuss below a SQUID design that outputs a current signal, and two AC designs which read out a voltage signal and a frequency signal, respectively.

\subsection{SQUID systems}

We first consider a circuit using a DC SQUID as the amplifier. The configuration is shown in Figure 2. The superconducting stripline, represented by $L_{k}$, is connected in parallel with the SQUID pick up coil, $L_{s q}$. The current in this circuit divides such that $I_{k} / I_{\nu q}=L_{s q} / L_{k}$. When a photon is absorbed in the calorimeter, there is a change in $L_{k}$ which is detected as a change in $I_{s q}$. The resulting current pulse, $\Delta V_{s q}$ is

$$
\Delta I_{s q}(E)=\frac{I_{b}}{L_{s q}}\left\{\frac{L_{k}(\mathrm{E})-L_{k_{o}}}{\left[1+\left(\frac{L_{k}(\mathrm{E})}{L_{s q}}\right]\right]\left[1+\left[\frac{L_{k_{o}}}{L_{s q}}\right)\right]}\right\} .
$$

where $L_{k_{o}}$ is the kinetic inductance at the base temperature, $T_{b}$, and $I_{b}$ is the bias current. $L_{k}(E)$ is the value of the kinetic inductance attained after the absorption of an $x$-ray of energy $E$. The base-line current, $I_{b}$, passing through the superconducting groundplane is chosen such that it will never exceed its critical value for any $x$-ray encountered.

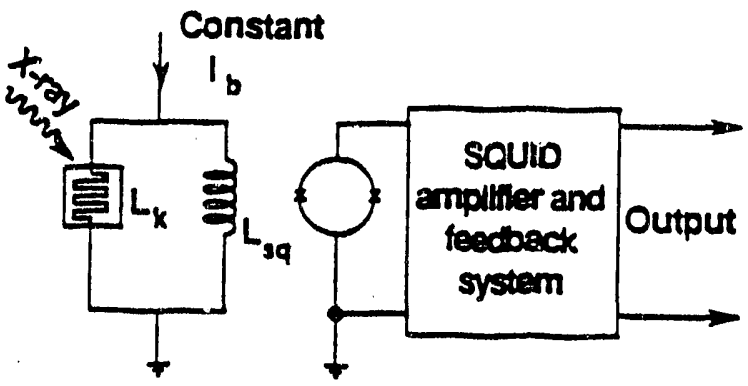

Figure 2. Kinetic inductance calorimeter in a SQUID amplifier circuit

For the devices considered here, the limiting current is $1 \mathrm{~mA}$. Given the way the current divides according to $L_{k}$ and $L_{s q}, I_{b}$ is set slightly above $1 \mathrm{~mA}$. Since the change in kinetic inductance is large, the gain of the transducing circuit is nonlineal; but can be calibrated. Note that this nonlinearity must be considered, as it is here, for any reasonable prediction of results.

Applying Equation 4 to the $3 \mathrm{eV}$ detector, and given a SQUID pick up coil inductance of $L_{s q}=2 \mu \mathrm{H}$, the largest signal obtainable at $10 \mathrm{keV}$ is approximately $0.2 \mathrm{~mA}$. This presents a problem for a commercial SQUID feedback system. The DC SQUID manufactured by Biomagnetic Technologies Incorporation (BTi), for example, has a maximum tracking speed of $100 \mathrm{~mA} / \mathrm{s}$. The current pulse from the $\mathrm{x}$-ray calorimeter (with a rise time of approximately $1 \mu \mathrm{s}$ ) has an initial rate of approximately $2 \times 10^{5} \mathrm{~mA} / \mathrm{s}$. Consequently, the SQUID will lose phase lock and produce significant measurement errors. 
A remedy to the loss of the phase lock problem is to slow down the signals with a thermal or electrical resistance. A weak thermal link between the absorber and the kinetic inductance thermometer could be constructed to slow down the temperature response of the thermometer. Alternatively, an electrical resistance in parallel with $L_{k}$ and $L_{s q}$ will also slow the rate of current change in the thermometer. The value of the resistor is chosen such that the steepest slope of the the filtered signal (the initial rise) corresponds to the maximum slew of thi SQUID (see Figure 3).

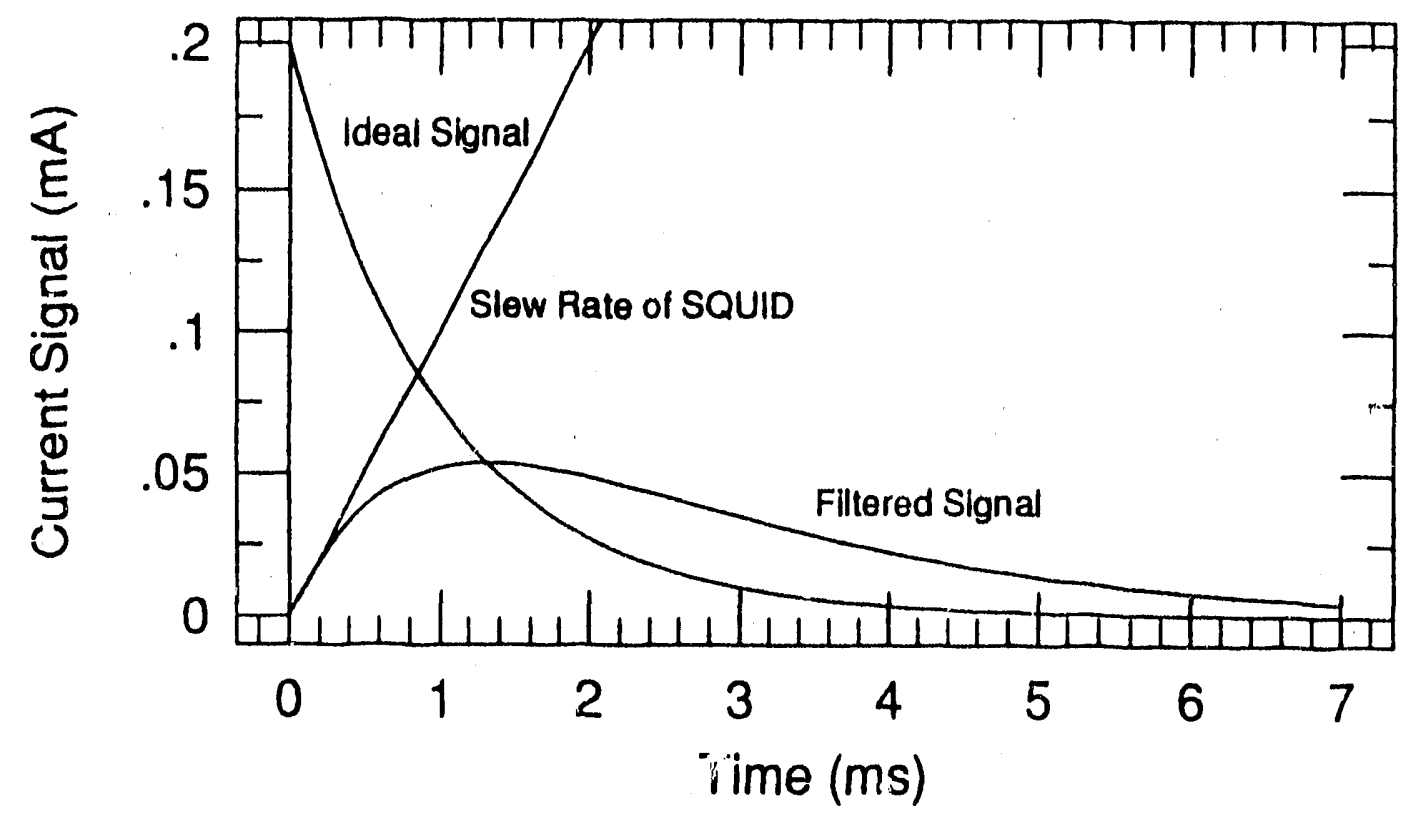

Figure 3. Effect of a resistive filter on the output signal.

A resistance on the order of $10^{-4} \Omega$ is sufficient to slow down the pulses and avoid loss of phase lock in the SQUID system. In this case, the thermometer continues to heat up at the original rate, but the SQUID only reads a fraction of the full amplitude of the thermal pulse profile. The signal to phonon noise is unaffected while the noise from the SQUID $\left(\delta E_{S Q U I D}=0.062 \mathrm{eV}\right)$ is still less than the phonon noise contribution $\left(\delta E_{\text {phonon }}=3 \mathrm{eV}\right)$. Here, we have assumed that the BTi DC SQUID has a flat noise spectrum of $1.5 \mathrm{pA} / \sqrt{\mathrm{Hz}}$ over a band width of $5000 \mathrm{~Hz}$.

To avoid the limitations of the commercial SQUID system, a customized SQUID system can be considered as a replacement. One possibility is to design a faster slew rate system where the tracking speed is fast enough to accommodate the fast $x$-ray pulses, and the signal does not have to be attenuated. ${ }^{10}$ In addition, the state of the art SQUIDs have, significantly lower noise. It may also be possible to include the kinetically inductive stripline inside the SQUID loop, instead of coupling it to the loop as shown in Figure 2. In this case, the signal will be indicated by a phase shift within the SQUID loop instead of a change in induced magnetic flux. By building the thermometer inside the amplifier, there would be no loss of signal due to the coupling to the SQUID. The design of such a detector, however, is outside of the scope of this study.

\subsection{FET configuration}

For this configuration we operate in a frequency range where the impedance of the stripline is large enough to generate a measurable voltage signal. The circuit is shown in Figure 4. 


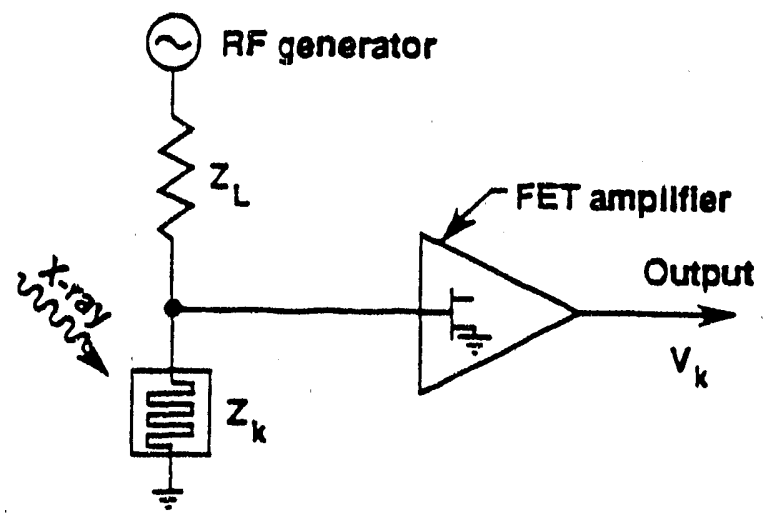

Figure 4. Circuit of a kinetic inductance calorimeter with an FET amplifier circuit.

A bias current is produced by connecting an RF generator in series with a load, $Z_{L}$. The voltage across the stripline is

$$
V_{K}(T)=V_{s} \frac{Z_{K}(T)}{Z_{K}(T)+Z_{L}}
$$

Here $V$, is the voltage level of the RF generator and $Z_{K}(T)$ is the impedance of the detector stripline:

$$
Z_{K}(T)=i \sqrt{\frac{L(T)}{C}} \tan (\omega \sqrt{L(T) C}),
$$

where $C$ is the total electrical capacitance between the stripline and the groundplane. The values of $Z_{L}$ and $V$, are chosen tw optimize the signal while maintaining current through the stripline below the critical current. For simplicity, in this example, we choose to make $Z_{L}$ a resistor, capacitive or inductiva loads could also be used. The Johnson noise generated by the resistor is smaller than the noise of the FET and therefore makes a negligible contribution to the total noise.

To optimize the signal to noise, the resonant nature of the stripline must be considered carefully. The inductance, $L(T)$, in the equation above is the total inductance as described in Equation 3. The operating frequency, $\omega$, is chosen so that the maximum change in inductance $\mathrm{L}$, will never cause the voltage signal to resonate. At the same time, the currents running through the stripline must not exceed the critical current of the superconducting material.

The optimal operating frequency for this detector is $4.2 \mathrm{MHz}$. Using a bandwidth of $5 \mathrm{kHz}$, and assuming that the dominant non-thermal noise source is derived from the FET amplifier, which has a flat noise spectrum of 2 $\mathrm{nV} / \sqrt{\mathrm{Hz}}$, the electronic contribution to the energy resolution is always less than $28 \mathrm{meV}$.

\subsection{Tunnel diode oscillator circuit}

A tunnel diode oscillator circuit has been used to measure kinetic inductance. ${ }^{11}$ For the oscillator circuit shown in Figure 5, the resonance frequency is

$$
\omega=\sqrt{(1 / L C)\left[1+\left(R_{s}^{2} C / L\right)\right]}
$$


When the photon is absorbed by the device, its temperature increases from $T_{\text {base }} 10 \mathrm{~T}(E)$, inducing a change in the kinetic inductance, thus altering the resonance frequency. The change in frequency can be detected using a phaselocked-loop detector. Given a high $\mathrm{Q}$, and incorporating the temperature dependence of $L$ into Equation 7 , the following frequency signal is obtained:

$$
\Delta \omega=\left(\frac{1}{\sqrt{L_{k}(\mathrm{E}) C}}-\frac{1}{\sqrt{L_{k_{o}} C}}\right)
$$

A detailed discussion of the theory of the tunnel diodes and their basic operations can be found in the literature. $^{12}$ The main feature to note is that witl ${ }_{\text {c }}$ careful biasing, the tunnel diode can operate as a low noise oscillator. The only sources of noise come from the residual resistance from the tank circuit (see Figure 5) and the voltage noise from the tunnel diode. ${ }^{13}$

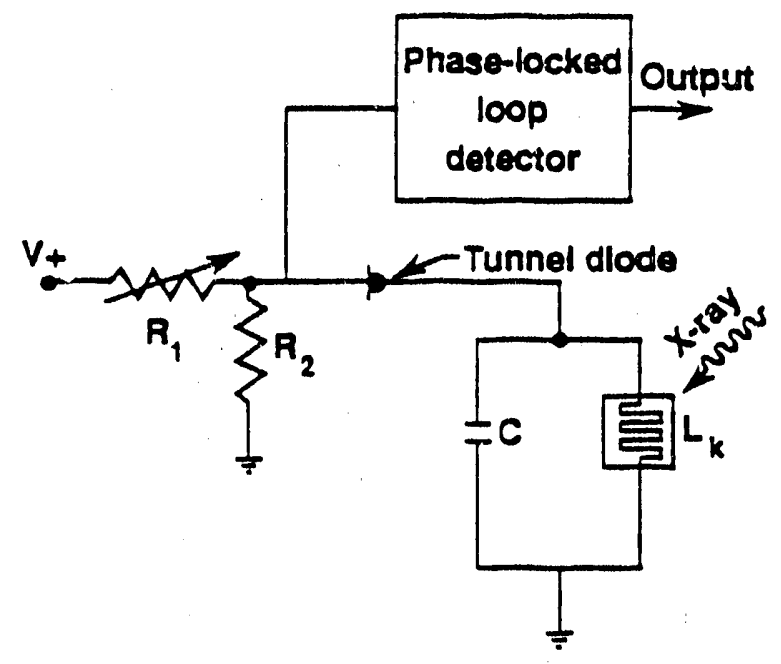

Figure 5. Tunnel diode oscillator circuit.

The tunnel diode noise is subdivided into Johnson and shot noise. Johnson noise originates from the series resistance, $R_{s}$, that varies according to the specific tunnel diode used. It has a typical value of a few viims. Numerically, for a typical diode operating at $15 \mathrm{MHz}$, the shot and Johnson noise are $1.7 \times 10^{-9} \mathrm{~V} / \sqrt{\mathrm{Hz}}$ and $5.0 \times 10^{-12} \mathrm{~V} / \sqrt{\mathrm{Hz}}$, respectively. Only the shot noise contributes.

To determine the electronic noise contribution for the tunnel diode oscillator circuit, we note that for any frequency $\omega$, the frequency noise, $\delta \omega$, is given by $\delta \omega / \omega(T)=\delta V / V$. Here, $\delta V$ is the voltage noise in the device and $V$ is the voltage amplitude of the oscillations. For a typical tunnel diode, $V$ is approximately $300 \mathrm{mV}$ and $\delta V$ dominated by the shot noise. The equivalent $\delta E_{\text {Diode }}$ for a $5 \mathrm{kHz}$ bandwidth should be below $7 \mathrm{meV}$ for a $3 \mathrm{eV}$ detector. Here, we have assumed that the phase-locked-loop would not contribute to the total noise.

\section{CONCLUSION}

In this paper, we have analyzed the potential performance of an $x$-ray calorimeter using a kinetic inductance thermometer. The overall results are presented in Table 2 below, and in Figure 6. 
TABLE 2

Contribution to Energy Resolution by Electronic Noise

Device

$3 \mathrm{eV}$ detector

\begin{tabular}{ll}
\hline BTi SQUID with Filter & $0.062 \mathrm{eV}$ \\
FET Amplifier Circuit & $0.028 \mathrm{eV}$ \\
Tunnel Diode & $0.007 \mathrm{eV}$ \\
\hline
\end{tabular}

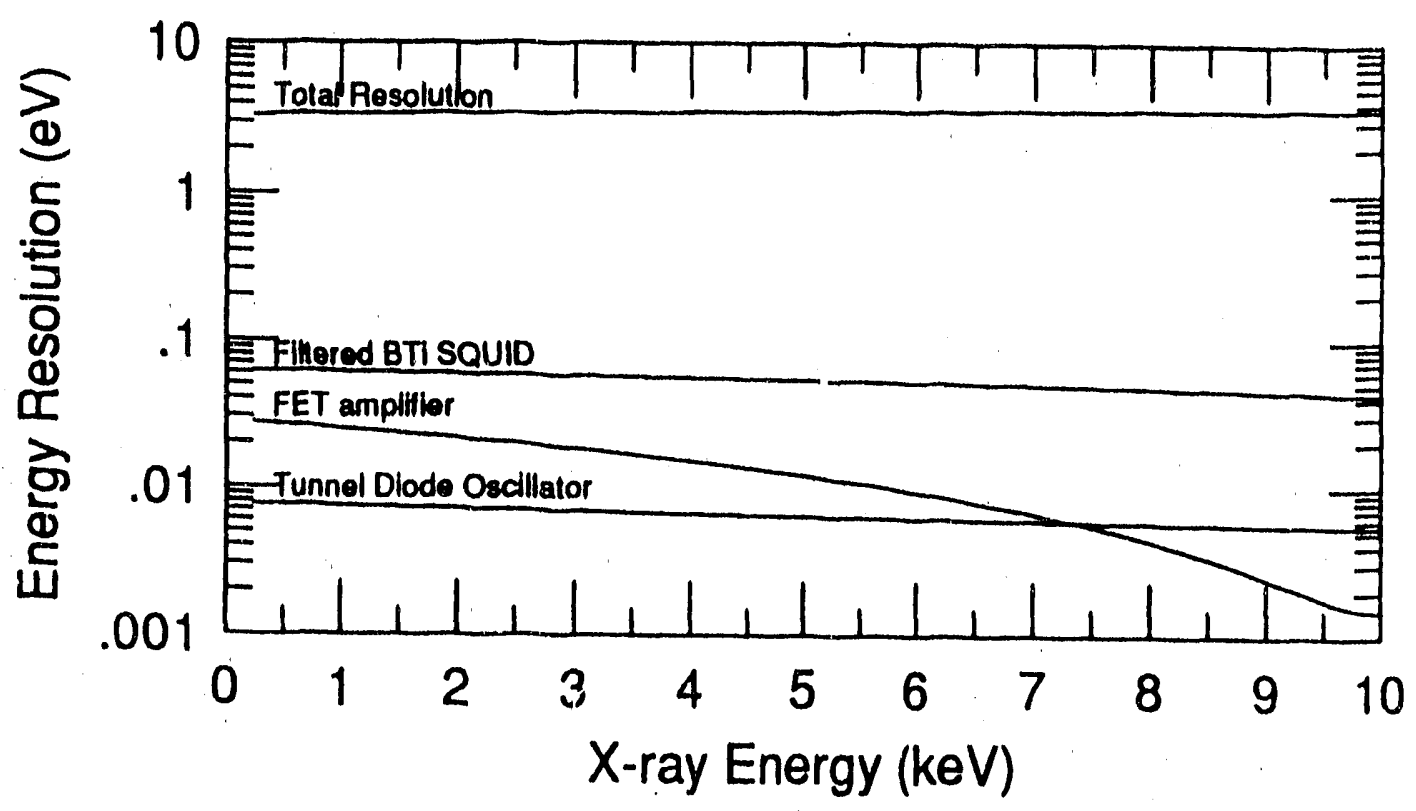

Figure 6. Energy resolution of each configuration over a $10 \mathrm{keV}$ range

In every case, the electronic noise is significantly below the $3 \mathrm{eV}$ phonon noise. The tunnel diode oscillator circuit proved, in theory, to be the best candidate since it gives the highest signal to electronic noise results. Despite the nonlinearities inherent in the FET circuit near the resonant frequency, the FET circuit is still a valid alternative. Sisise the FET configuration is similar to that used in the resistive calorimetry systems, it can be easily implemented into existing resistive systems and tested once the device is fabricated. Although the commercial SQUIDs have an intrinsic slew rate limitation, their non-thermal noise contribution to the total is still negligible. Since the configurations with the lowest electronic noise are most likely to produce phonon noise limited results, all three designs considered here will have the possibility of out-performing the existing resistive calorimeters; therefore, all are likely candidates as thermometers in the actual fabrication of the device. In particular, the FET and tunnel diode methods offer simple and promising altematives to SQUID systems for measuring kinetic inductance.

\section{ACKNOWLEDGEMENT}

We wish to thank John Clarke and Paul Richards for invaluable information. Special thanks to Ricky Tang and Bryce Yan for assistance in the graphics and lay-out of this paper. This work was performed under the auspices of the US Department of Energy by Lawrence Livermore National Laboratory under Contract No. W. 7405-ENG-48. 


\section{REFERENCES}

1. S. H. Mosley, J. C. Mather and D. McCommon, "Thermal detectors as x-ray spectrometers," J. Appl. Phys 56, 1257-1262, 1984.

2. E. H. Silver, S. E. Labov, F. Goulding, N. Madden, D. Landis, J. Beeman, T. Pfafman, L. Melkonian, I. Millett, and $Y$. Wai, "Hizh resolution $x$-ray spectroscopy using germanium microcalorimeters," Proc. SPIE 1159, 423-432, 1989.

3. D. McCammon, B. Edwards, M. Juda, P. Plucinsky, J. Zhang, R. Kelly, S. Holt, G. Modejski, S. Moseley and A. Szymkowiak, "Thermal Detection of X-rays" in Low Temperature Detectors for Neutrinos and Dark Matter III (Edition Frontieres: Gif Sar Yevett Cedes), 1989.

4. T. Pfafman, E. H. Silver, S. E. Labov, J. Beeman, F. S. Goulding, W. L. Hansen, D. Landis, N. W. Madden, "Test results of a prototype dielectric microcalorimeter," Proc SPIE 1344, 1990 (this volume).

5. R. L. Kautz, "Picosecond Pulses on Supercondiucting Striplines", J. Appl. Phys. 49, 308-314, 1978.

6. D. G. McDonald, "Novel Superconducting Thermometer for Bolometric Applications" Appl. Phys. Lett. 50, 775-777, 1987.

7. G. L. Rawley, R. L. Kelley, S. H. Moseley, and A. E. Szymkowiak, "Progress in kinetic inductane thermometers for $\mathrm{x}$-ray calorimeters," Proc. SPIE 1159, 414-422, 1989.

8. A. K. Raychardhuri, C. Egloff, and L. Rinderer, "Electrodynamics of Superconductors. Response to a Weak, Low-Frequency Magnetic Field" J. Low Temp. Phys. 53, 513-561, 1983.

9 D. U. Gubser and R. J. Soulen, Jr, "Thermodynamic Properties of Superconducting Iridium", J. of Low Temperature Phys, 13, 211-226, 1973.

10. J. Clarke, Chapter in Proceedings of NATO. Advanced Study Institute on Superconductive electronics, P. 87, M. Nisenoff and H. Weinstock (eds.) 1989.

11. R. Meservey and P. M. Tedrow, "Measurements of the Kinetic Inductance of Superconducting Linear Structures", J. of Appl. Phys., 40, 2028-2034, 1969.

12. S. P. Gentile, Basic Theory and Application of Tunnel Diodes, D. Van Nostrand Company, Inc., Princeton, New Jersey, 1962.

13. J. J. Tiemann, "Shot Noise in Tunnel Diode Amplifiers", Proceedings of the IRE , 48, 1418-1423, 1960. 

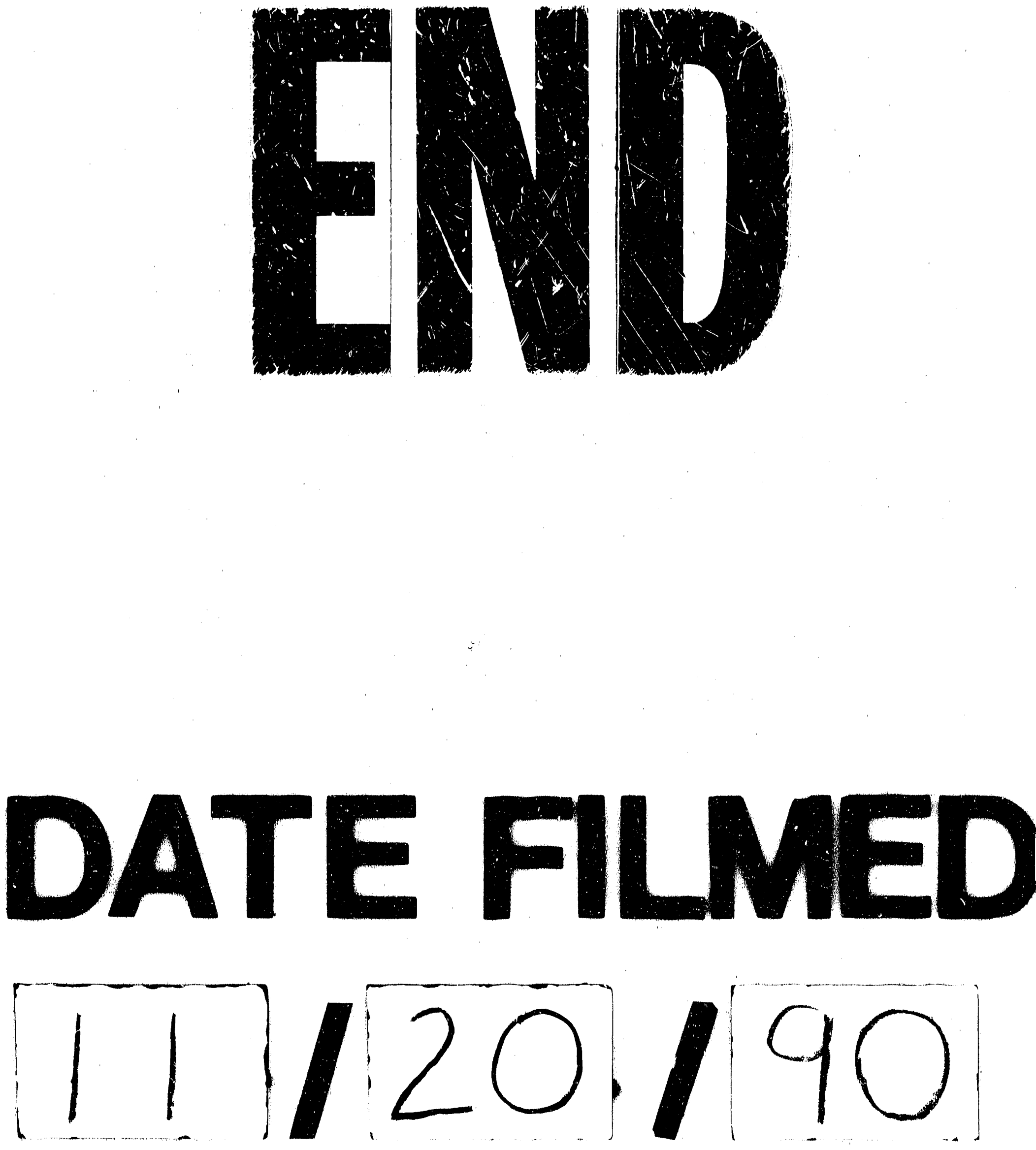